\title{
Commuting Toeplitz and Hankel Operators on Harmonic Dirichlet Spaces
}

\author{
Qian Ding, ${ }^{1}$ Yong Chen, ${ }^{2}$ and Yufeng $\mathrm{Lu}^{1}$ \\ ${ }^{1}$ School of Mathematical Sciences, Dalian University of Technology, Dalian, Liaoning 116024, China \\ ${ }^{2}$ Department of Mathematics, Zhejiang Normal University, Jinhua, Zhejiang 321004, China \\ Correspondence should be addressed to Yong Chen; ychen227@gmail.com
}

Received 3 February 2017; Accepted 5 March 2017; Published 13 April 2017

Academic Editor: Kehe Zhu

Copyright (c) 2017 Qian Ding et al. This is an open access article distributed under the Creative Commons Attribution License, which permits unrestricted use, distribution, and reproduction in any medium, provided the original work is properly cited.

On the harmonic Dirichlet space of the unit disk, the commutativity of Toeplitz and Hankel operators is studied. We obtain characterizations of commuting Toeplitz and Hankel operators and essentially commuting (semicommuting) Toeplitz and Hankel operators with general symbols.

\section{Introduction}

Let $\mathbb{D}$ be the open unit disk in the complex plane $\mathbb{C}$ and $d A$ denote the normalized area measure on $\mathbb{D}$. The Sobolev space $\mathcal{S}$ is the completion of the space of all smooth functions $f$ on $\mathbb{D}$ with norm

$$
\begin{aligned}
\|f\| & =\left\{\left|\int_{\mathbb{D}} f d A\right|^{2}+\int_{\mathbb{D}}\left(\left|\frac{\partial f}{\partial z}\right|^{2}+\left|\frac{\partial f}{\partial \bar{z}}\right|^{2}\right) d A\right\}^{1 / 2} \\
& <\infty
\end{aligned}
$$

and the inner product of the Sobolev space $\mathcal{S}$ is

$$
\begin{aligned}
\langle f, g\rangle= & \int_{\mathbb{D}} f d A \int_{\mathbb{D}} \bar{g} d A+\left\langle\frac{\partial f}{\partial z}, \frac{\partial g}{\partial z}\right\rangle_{2} \\
& +\left\langle\frac{\partial f}{\partial \bar{z}}, \frac{\partial g}{\partial \bar{z}}\right\rangle_{2},
\end{aligned}
$$

where $\langle\cdot, \cdot\rangle_{2}$ denotes the inner product in the Hilbert space $L^{2}(\mathbb{D}, d A)$.

The Dirichlet space $\mathscr{D}$ is the closed subspace of $\delta$ consisting of all holomorphic functions on $\mathbb{D}$ vanishing at zero and the harmonic Dirichlet space $\mathscr{D}_{h}$ is the closed subspace of $\mathcal{S}$ consisting of all harmonic functions on $\mathbb{D}$. There is the relation that $\mathscr{D}_{h}=\mathscr{D} \oplus \mathbb{C} \oplus \overline{\mathscr{D}}$, where $\overline{\mathscr{D}}=\{\bar{f}$ : $f \in \mathscr{D}\}$. It is well known that each point evaluation in $\mathscr{D}_{h}$ is a bounded linear functional on $\mathbb{D}$, so, for every $z \in \mathbb{D}$, there exists a unique function $R_{z} \in \mathscr{D}_{h}$ which has the reproducing property

$$
f(z)=\left\langle f, R_{z}\right\rangle
$$

for every $f \in \mathscr{D}_{h}$.

Since $\mathscr{D}_{h}=\mathscr{D} \oplus \mathbb{C} \oplus \overline{\mathscr{D}}$, there is a relation

$$
R_{z}=K_{z}+\overline{K_{z}}+1
$$

where $K_{z}$ is the reproducing kernel for Dirichlet space $\mathscr{D}$ and is given by

$$
K_{z}(w)=-\ln (1-\bar{z} w)=\sum_{k=1}^{\infty} \frac{\bar{z}^{k} w^{k}}{k} .
$$

Let $P$ denote the orthogonal projection of $\mathcal{S}$ onto $\mathscr{D}$ and $Q$ denote the orthogonal projection of $\mathcal{S}$ onto $\mathscr{D}_{h}$. Since $\operatorname{Pg}(z)=$ $\left\langle g, K_{z}\right\rangle$ for $g \in \mathcal{S}$ and $z \in \mathbb{D}$, then by (4) it is easy to see that

$$
Q(g)=P(g)+\overline{P(\bar{g})}+\langle g, 1\rangle
$$

for any function $g \in \mathcal{S}$.

For a function $\varphi \in \mathcal{S}$, the Toeplitz operator $T_{\varphi}: \mathscr{D}_{h} \rightarrow$ $\mathscr{D}_{h}$ with the symbol $\varphi$ is densely defined by

$$
T_{\varphi}(f)=Q(\varphi f)
$$


for $f \in \mathscr{D}_{h}$ and $\varphi f \in \mathcal{S}$. The (small) Hankel operator $\Gamma_{\psi}$ : $\mathscr{D}_{h} \rightarrow \mathscr{D}_{h}$ with the symbol $\psi$ is densely defined by

$$
\Gamma_{\psi}(g)=J Q(\psi g)
$$

for $g \in \mathscr{D}_{h}$ and $\psi g \in \mathcal{S}$, where $J$ is an unitary operator defined by $J f(z)=f(\bar{z})$ for $f \in \mathcal{S}$. It is easy to check that $Q J=J Q$, so Hankel operator has the relation with the Toeplitz operator as follows:

$$
\Gamma_{\psi}=J T_{\psi}=T_{J \psi} J .
$$

It follows that $\Gamma_{1}=J$ since $T_{1}=I$, the identity operator.

On the classical Hardy space, Brown and Halmos [1] showed the necessary and sufficient conditions for Toeplitz operator which has the commutativity properties. Also, they obtained the characterization for the product problem of the Toeplitz operators. Their works have been generalized onto the case on the (harmonic) Bergman or Dirichlet space by many authors; see [2-6] and the references therein. Many works related to the product involving Toeplitz or Hankel operators are referred to in [7-12].

In recent years, Chen et al. have studied the algebraic properties of Toeplitz operators on the harmonic Dirichlet space ([13]) with general symbols. Later, Feng et al. studied the commutativity of Toeplitz operator and Hankel operator, or two Hankel operators on harmonic Dirichlet space ([14, $15]$ ), and they focused on the operators with harmonic symbols.

In the present paper, we continue to study the same characterizing problems for general symbols. In order to handle the general symbols, in the second section, we will give a characterization for when the sum of products of two Toeplitz operators equals a Hankel operator, which is the key to prove our main results (see Proposition 5). In the third section, we give the commutativity of Toeplitz and Hankel operators (see Theorem 10) or two Hankel operators (see Theorem 11). We also characterize when the product of two Hankel operators equals another Hankel operator (see Theorem 12), and then, as an consequence, we get the semicommutativity of two Hankel operators (see Corollary 13). In the last section, we study the essential (semi)commutativity of Toeplitz and Hankel operators or two Hankel operators.

\section{Preliminaries}

For $\varphi \in \mathcal{S}$, it turns out that $\varphi\left(r e^{i \theta}\right)$ is absolutely continuous on $r \in[0,1)$ for almost every $\theta \in[0,2 \pi]$ and absolutely continuous on $\theta \in[0,2 \pi]$ for almost every $r \in[0,1)$. In particular, the radial limit $\left.\varphi\right|_{\partial \mathbb{D}}:=\lim _{r \rightarrow 1} \varphi\left(r e^{i \theta}\right)$ exists for almost every $\theta \in[0,2 \pi]$. Moreover, we have $\left.\varphi\right|_{\partial \mathbb{D}} \in L^{1}(\partial \mathbb{D})$, the space of integrable functions on $\partial \mathbb{D}$, and the Poisson extension of $\left.\varphi\right|_{\partial \mathbb{D}}$ belongs to $\mathcal{S}$. See $[16,17]$ for details and related facts.

A nonnegative Borel measure $\mu$ on $\mathbb{D}$ is called a $\mathscr{D}$ Carleson measure if there exists a constant $C>0$ such that

$$
\int_{\mathbb{D}}|f|^{2} d \mu \leq C\|f\|^{2}
$$

for every $f \in \mathscr{D}$. See $[18,19]$ for the details. We let $\mathscr{M}$ be the space of all $U \in \mathcal{S}$ for which $u$ is bounded measurable on $\mathbb{D}$ and $|\partial u / \partial z|^{2} d A$ and $|\partial u / \partial \bar{z}|^{2} d A$ are $\mathscr{D}$-Carleson measures, where $u$ is the Poisson extension of $\left.U\right|_{\partial \mathbb{D}}$.

It is known that $T_{\varphi}$ is bounded on the harmonic Dirichlet space $\mathscr{D}_{h}$ if and only if $\varphi \in \mathscr{M}$ (see [13]), so, by (9), $\Gamma_{\varphi}$ is bounded on $\mathscr{D}_{h}$ if and only if $\varphi \in \mathscr{M}$.

We let

$$
\Delta_{0}=\left\{\varphi \in \mathcal{S}:\left.\varphi\right|_{\partial \mathbb{D}}=0\right\} .
$$

It is easy to see $\phi \Delta_{0} \subset \Delta_{0}$ when $\phi f \in \mathcal{S}$ for each $f \in \mathcal{S}$, and also $\phi \in \Delta_{0}$ if and only if $\phi=0$ when $\phi \in \mathscr{D}_{h}$. Moreover, a decomposition for the Sobolev space $\mathcal{S}$ proved in $[17,20]$ gives the notion that

$$
\mathcal{S}=\left(\Delta_{0}+\mathbb{C}\right) \oplus \mathscr{D} \oplus \overline{\mathscr{D}} .
$$

We start with the following lemma showing that the boundary vanishing property of a symbol gives a simple behavior of the corresponding Toeplitz operator (see [13]).

Lemma 1. Let $\varphi \in \Delta_{0}$. Then, one has

$$
T_{\varphi} f=\int_{\mathbb{D}} \varphi f d A
$$

for every polynomial $f \in \mathscr{D}_{h}$. In particular, $T_{\varphi}$ can be extended to a bounded linear functional on $\mathscr{D}_{h}$.

Note that Lemma 1 shows that, for $\varphi \in \Delta_{0}, T_{\varphi}$ is at most rank one. It is also the same case for $\Gamma_{\varphi}$ when $\varphi \in \Delta_{0}$ by relation (9). In addition, $P T_{\varphi} P$ is the Toeplitz operator on $\mathscr{D}$, denoted by $\widetilde{T}_{\varphi}$, and thus, for $\varphi \in \mathscr{M} \cap \mathscr{D}_{h}$, the compactness of $\widetilde{T}_{\varphi}$ implies $\varphi=0$ (see $[16,17]$ ), so, by (9) and Lemma 1, we have

$$
\Gamma_{\varphi} \text { is compact } \Longleftrightarrow T_{\varphi} \text { is compact } \Longleftrightarrow \varphi \in \Delta_{0} .
$$

We also need the following result.

Lemma 2. Let $\varphi, \psi \in \mathscr{M}$; then the following statements are equivalent:
(a) $\Gamma_{\psi}-T_{\varphi}$ is compact.
(b) $\Gamma_{\psi}-T_{\varphi}$ is finite rank.
(c) $\varphi, \psi \in \Delta_{0}$.

Proof. Let $\varphi=\varphi_{0}+\varphi_{1}$ and $\psi=\psi_{0}+\psi_{1}$; here, $\varphi_{0}, \psi_{0} \in \Delta_{0}$ and $\varphi_{1}, \psi_{1} \in \mathscr{D}_{h}$. Note that, by Lemma $1, \Gamma_{\psi_{0}}$ and $T_{\varphi_{0}}$ are finite rank operators, so we only need to show that $\Gamma_{\psi_{1}}-T_{\varphi_{1}}$ is compact or finite rank if and only if $\varphi_{1}=\psi_{1}=0$.

If $\Gamma_{\psi_{1}}-T_{\varphi_{1}}$ is compact, then $P\left(\Gamma_{\psi_{1}}-T_{\varphi_{1}}\right) P$ is compact; that is, $\widetilde{\Gamma}_{\psi_{1}}-\widetilde{T}_{\varphi_{1}}=K$; here, $\widetilde{\Gamma}_{\psi_{1}}=P \Gamma_{\psi_{1}} P$ is the (small) Hankel operator on the Dirichlet space $\mathscr{D}($ see $[17,21]), \widetilde{T}_{\varphi_{1}}=P T_{\varphi_{1}} P$ is the Toeplitz operator on $\mathscr{D}$, and $K$ is a compact operator on D.

Claim. $\widetilde{T}_{\bar{z}} \widetilde{\Gamma}_{\psi_{1}}=\widetilde{\Gamma}_{\psi_{1}} \widetilde{T}_{z}$.

In fact, it is easy to check

$$
P\left[\bar{z}^{m} z^{n}\right](z)= \begin{cases}z^{n-m}, & n>m, \\ 0, & n \leq m .\end{cases}
$$


Let $\psi_{1}=\psi_{1}(0)+\sum_{j=1}^{\infty}\left(b_{j} z^{j}+b_{-j} \bar{z}^{j}\right)$. Then, for $n \in \mathbb{Z}_{+}$,

$$
P J\left(\psi_{1} z^{n}\right)=\sum_{j>n} b_{-j} z^{j-n}
$$

by (15). So, for positive integers $n$ and $m$, we have

$$
\begin{aligned}
\left\langle\widetilde{T}_{\bar{z}} \widetilde{\Gamma}_{\psi_{1}} z^{n}, z^{m}\right\rangle & =\left\langle\bar{z} P J\left(\psi_{1} z^{n}\right), z^{m}\right\rangle \\
& =\sum_{j>n} b_{-j}\left\langle z^{j-n} \bar{z}, z^{m}\right\rangle \\
& =\sum_{j>n} b_{-j}(j-n) m\left\langle z^{j-n-1} \bar{z}, z^{m-1}\right\rangle_{2} \\
& =b_{-n-m-1} m, \\
\left\langle\widetilde{\Gamma}_{\psi_{1}} \widetilde{T}_{z^{n}} z^{n}, z^{m}\right\rangle & =\left\langle J\left(\psi_{1} z^{n+1}\right), z^{m}\right\rangle \\
& =\sum_{j=1}^{\infty} b_{-j}\left\langle z^{j} \bar{z}^{n+1}, z^{m}\right\rangle \\
& =\sum_{j=1}^{\infty} b_{-j} j m\left\langle z^{j-1} \bar{z}^{n+1}, z^{m-1}\right\rangle_{2} \\
& =b_{-n-m-1} m,
\end{aligned}
$$

and hence the claim holds.

Since $\widetilde{\Gamma}_{\psi_{1}}=\widetilde{T}_{\varphi_{1}}+K$, by the claim, we get

$$
\widetilde{T}_{\bar{z}} \widetilde{T}_{\varphi_{1}}+\widetilde{T}_{\bar{z}} K=\widetilde{T}_{\varphi_{1}} \widetilde{T}_{z}+K \widetilde{T}_{z}
$$

It is well known that $\widetilde{T}_{\bar{z}} \widetilde{T}_{\varphi_{1}}=\widetilde{T}_{\bar{z} \varphi_{1}}$ and $\widetilde{T}_{\varphi_{1}} \widetilde{T}_{z}=\widetilde{T}_{z \varphi_{1}}$ (see $[6,17,20])$; then, by the above equality, we get

$$
\widetilde{T}_{\bar{z} \varphi_{1}-z \varphi_{1}}=K \widetilde{T}_{z}-\widetilde{T}_{\bar{z}} K
$$

which gives $\bar{z} \varphi_{1}-z \varphi_{1} \in \Delta_{0}$, so $\varphi_{1}=0$ because $\bar{z}-z \neq 0$ on the boundary of $\mathbb{D}$ except at $z= \pm 1$. Thus, we see that $\Gamma_{\psi_{1}}$ is a compact operator which gives $\psi_{1}=0$ by (14). $\psi_{1}=0$.

If $\Gamma_{\psi_{1}}-T_{\varphi_{1}}$ is finite rank, then similar arguments give $\varphi_{1}=$

The sufficiency is obvious. The proof is complete.

We let $\mathscr{P}$ denote the set of all $U \in \mathcal{S}$ such that for all integers $n \geq 0$

$$
\begin{aligned}
& \int_{\mathbb{D}} U \bar{w}^{n} d A=(n+1) \int_{\mathbb{D}} u \bar{w}^{n} d A, \\
& \int_{\mathbb{D}} U w^{n} d A=(n+1) \int_{\mathbb{D}} u w^{n} d A,
\end{aligned}
$$

where $u$ is the Poisson extension of $\left.U\right|_{\partial \mathbb{D}}$. Note that, for harmonic function $U \in \mathcal{S}$, we can check that $U \in \mathscr{P}$ if and only if $U$ is constant. Also, for $U \in \mathscr{M}$, by (9) and Lemma 1 we see that $T_{U}=0$ if and only if $\Gamma_{U}=0$ and if and only if $U \in \Delta_{0} \cap \mathscr{P}$. Now, by Lemmas 1 and 2 , we can get easily the following result which has independent interest.

Corollary 3. Let $\varphi, \psi \in \mathscr{M}$. Then, $\Gamma_{\psi}=T_{\varphi}$ if and only if $\varphi, \psi \in$ $\Delta_{0}$ and $\psi-\varphi \in \mathscr{P}$.

Let $U, V \in \mathscr{M}$ and $u, v$ are the Poisson extensions of $\left.U\right|_{\partial \mathbb{D}}$ and $\left.V\right|_{\partial \mathbb{D}}$, respectively. Then, it is easy to see $U-u, V-v \in \Delta_{0}$. Fix a polynomial $f \in \mathscr{D}_{h}$. By Lemma 1 , we have

$$
\begin{aligned}
T_{V} f & =T_{v} f+\int_{\mathbb{D}}(V-v) f d A, \\
Q(U) & =T_{U} 1=T_{u} 1+\int_{\mathbb{D}}(U-u) d A \\
& =u+\int_{\mathbb{D}}(U-u) d A .
\end{aligned}
$$

It follows from Lemma 1 again that

$$
\begin{aligned}
T_{U} T_{V} f= & T_{U} T_{v} f+Q(U) \int_{\mathbb{D}}(V-v) f d A \\
= & T_{u} T_{v} f+\int_{\mathbb{D}}(U-u) T_{v} f d A \\
& +Q(U) \int_{\mathbb{D}}(V-v) f d A \\
= & T_{u} T_{v} f+\int_{\mathbb{D}}(U-u) T_{v} f d A \\
& +u \int_{\mathbb{D}}(V-v) f d A \\
& +\int_{\mathbb{D}}(U-u) d A \int_{\mathbb{D}}(V-v) f d A .
\end{aligned}
$$

So,

$$
\begin{aligned}
& T_{U} T_{V} f-T_{U} T_{V} f(0) \\
& =T_{u} T_{v} f-T_{u} T_{v} f(0) \\
& \quad+[u-u(0)] \int_{\mathbb{D}}(V-v) f d A .
\end{aligned}
$$

The above will be used to characterize when the following product of Toeplitz operators equals a Hankel operator:

$$
\sum_{l=1}^{N} T_{U_{l}} T_{V_{l}}=\Gamma_{H}
$$

for $U_{l}, V_{l}, H \in \mathscr{M}(1 \leq l \leq N)$. Here, $N$ is a fixed positive integer. To this end, we also need the following lemma which is easy to verify by (6) (see Lemmas 3.1 and 4.2 in [13] for the details). 
Lemma 4. Let $u, v \in \mathscr{M}$ be harmonic and write

$$
\begin{aligned}
& u(z)=\sum_{k=0}^{\infty} a_{k} z^{k}+\sum_{k=1}^{\infty} a_{-k} \bar{z}^{k}, \\
& v(z)=\sum_{k=0}^{\infty} b_{k} z^{k}+\sum_{k=1}^{\infty} b_{-k} \bar{z}^{k}
\end{aligned}
$$

for the power series expansions of $u, v$, respectively. Then, one has

$$
\begin{aligned}
& T_{u} T_{v}\left[z^{n}\right](z)-T_{u} T_{v}\left[z^{n}\right](0) \\
& \quad=T_{u v}\left[z^{n}\right](z)-T_{u v}\left[z^{n}\right](0)-\frac{n b_{-n}[u-u(0)]}{n+1}, \\
& T_{u} T_{v}\left[\bar{z}^{n}\right](z)-T_{u} T_{v}\left[\bar{z}^{n}\right](0) \\
& \quad=T_{u v}\left[\bar{z}^{n}\right](z)-T_{u v}\left[\bar{z}^{n}\right](0)-\frac{n b_{n}[u-u(0)]}{n+1}
\end{aligned}
$$

for every integer $n \geq 0$ and $z \in \mathbb{D}$.

We now give the following necessary conditions for the sum of products of two Toeplitz operators equal to a Hankel operator which is the key to characterize the related problems.

Proposition 5. Let $U_{l}, V_{l}, H \in \mathscr{M}$ and $u_{l}, v_{l}, h$ be the Poisson extensions of $\left.U_{l}\right|_{\partial \mathbb{D}},\left.V_{l}\right|_{\partial \mathbb{D}},\left.H\right|_{\partial \mathbb{D}}$, respectively, $1 \leq l \leq N$. Suppose $\sum_{l=1}^{N} T_{U_{l}} T_{V_{l}}=\Gamma_{H}$. Then, $\sum_{l=1}^{N} u_{l} v_{l} \in \Delta_{0}$ and $h=0$. Moreover, if there is $l_{0}$ such that $V_{l_{0}} \notin \mathscr{P}$, then

$$
u_{l_{0}}=\sum_{l \neq l_{0}} \alpha_{l} u_{l}+\beta
$$

for some constants $\alpha_{l}\left(1 \leq l \leq N, l \neq l_{0}\right)$ and some constant $\beta$.

Proof. Consider power series expansions of $u_{l}, v_{l}$ as

$$
\begin{aligned}
& u_{l}(z)=\sum_{k=0}^{\infty} a_{k}^{(l)} z^{k}+\sum_{k=1}^{\infty} a_{-k}^{(l)} \bar{z}^{k} \\
& v_{l}(z)=\sum_{k=0}^{\infty} b_{k}^{(l)} z^{k}+\sum_{k=1}^{\infty} b_{-k}^{(l)} \bar{z}^{k}
\end{aligned}
$$

for $1 \leq l \leq N$. By (23) and Lemma 4, for every integer $n \geq 0$, we have

$$
\begin{aligned}
& T_{U_{l}} T_{V_{l}} z^{n}-T_{U_{l}} T_{V_{l}} z^{n}(0) \\
& =T_{u_{l}} T_{v_{l}} z^{n}-T_{u_{l}} T_{v_{l}} z^{n}(0) \\
& \quad+\left[u_{l}-u_{l}(0)\right] \int_{\mathbb{D}}\left(V_{l}-v_{l}\right) z^{n} d A
\end{aligned}
$$

$$
\begin{aligned}
= & T_{u_{l} v_{l}} z^{n}-T_{u_{l} v_{l}} z^{n}(0)-\frac{n b_{-n}^{(l)}\left[u_{l}-u_{1}(0)\right]}{n+1} \\
& +\left[u_{l}-u_{l}(0)\right] \int_{\mathbb{D}}\left(V_{l}-v_{l}\right) z^{n} d A \\
= & T_{u_{l} v_{l}} z^{n}-T_{u_{l} v_{l}} z^{n}(0) \\
& +\left[u_{l}-u_{l}(0)\right]\left(\int_{\mathbb{D}} V_{l} z^{n} d A-b_{-n}^{(l)}\right) ;
\end{aligned}
$$

here in the last equality we have used the identity

$$
\int_{\mathbb{D}} v_{l} z^{n} d A=\frac{b_{-n}^{(l)}}{n+1}
$$

for each $n$ and $l$. On the other hand, for each nonnegative integer $n$,

$$
\Gamma_{H} z^{n}-\Gamma_{H} z^{n}(0)=\Gamma_{h} z^{n}-\Gamma_{h} z^{n}(0)
$$

since $H-h \in \Delta_{0}$. So, from $\sum_{l=1}^{N} T_{U_{l}} T_{V_{l}}=\Gamma_{H}$ and (29), we get

$$
\begin{array}{r}
\left(T_{\sum_{l=1}^{N} u_{l} v_{l}}-\Gamma_{h}\right) z^{n}-\left(T_{\sum_{l=1}^{N} u_{l} v_{l}}-\Gamma_{h}\right) z^{n}(0) \\
=-\sum_{l=1}^{N}\left[u_{l}-u_{l}(0)\right]\left(\int_{\mathbb{D}} V_{l} z^{n} d A-b_{-n}^{(l)}\right)
\end{array}
$$

for integer $n \geq 0$.

Similarly, we consider $T_{U_{l}} T_{V_{l}} \bar{z}^{n}-T_{U_{l}} T_{V_{l}} \bar{z}^{n}(0)$ as done in (29) to get

$$
\begin{aligned}
& T_{U_{l}} T_{V_{l}} \bar{z}^{n}-T_{U_{l}} T_{V_{l}} \bar{z}^{n}(0) \\
& =T_{u_{l}} T_{v_{l}} \bar{z}^{n}-T_{u_{l}} T_{v_{l}} \bar{z}^{n}(0) \\
& \quad+\left[u_{l}-u_{l}(0)\right] \int_{\mathbb{D}}\left(V_{l}-v_{l}\right) \bar{z}^{n} d A \\
& =T_{u_{l} v_{l}} \bar{z}^{n}-T_{u_{l} v_{l}} \bar{z}^{n}(0) \\
& \quad+\left[u_{l}-u_{l}(0)\right]\left(\int_{\mathbb{D}} V_{l} \bar{z}^{n} d A-b_{n}^{(l)}\right),
\end{aligned}
$$

so similarly we can get the identity

$$
\begin{array}{r}
\left(T_{\sum_{l=1}^{N} u_{l} v_{l}}-\Gamma_{h}\right) \bar{z}^{n}-\left(T_{\sum_{l=1}^{N} u_{l} v_{l}}-\Gamma_{h}\right) \bar{z}^{n}(0) \\
=-\sum_{l=1}^{N}\left[u_{l}-u_{l}(0)\right]\left(\int_{\mathbb{D}} V_{l} \bar{z}^{n} d A-b_{n}^{(l)}\right)
\end{array}
$$

for integer $n \geq 0$.

It follows from (32) and (34) that $T_{\sum_{l=1}^{N} u_{l} v_{l}}-\Gamma_{h}$ is a finite rank operator, and thus Lemma 2 gives $h=0$ and $\sum_{l=1}^{N} u_{l} v_{l} \epsilon$ $\Delta_{0}$, and by Lemma 1 the latter one is

$$
T_{\sum_{l=1}^{N} u_{l} v_{l}} \varphi-T_{\sum_{l=1}^{N} u_{l} v_{l}} \varphi(0)=0
$$


for each $\varphi \in \mathscr{D}_{h}$. It follows that the left sides of identities (32) and (34) are both zero for each integer $n \geq 0$, and so are the right sides of these two identities; that is,

$$
\begin{aligned}
& \sum_{l=1}^{N}\left[u_{l}-u_{l}(0)\right]\left(\int_{\mathbb{D}} V_{l} z^{n} d A-b_{-n}^{(l)}\right)=0, \\
& \sum_{l=1}^{N}\left[u_{l}-u_{l}(0)\right]\left(\int_{\mathbb{D}} V_{l} \bar{z}^{n} d A-b_{n}^{(l)}\right)=0
\end{aligned}
$$

for each integer $n \geq 0$. If $V_{l_{0}} \notin \mathscr{P}$, then there is integer $n_{0} \geq 0$ such that

$$
\begin{array}{r}
\int_{\mathbb{D}} V_{l_{0}} z^{n_{0}} d A-b_{-n_{0}}^{\left(l_{0}\right)} \neq 0, \\
\text { or } \int_{\mathbb{D}} V_{l_{0}} \bar{z}^{n_{0}} d A-b_{n_{0}}^{\left(l_{0}\right)} \neq 0,
\end{array}
$$

and hence (36) or (37) gives (27), as desired. The proof is complete.

\section{Commutativity of Toeplitz and Hankel Operators}

As one application of Proposition 5, we have the following result.

Proposition 6. Let $u_{1}, u_{2}, v_{1}, v_{2} \in \mathscr{M} \cap \mathscr{D}_{h}$. Then, $T_{u_{1}} T_{v_{1}}=$ $T_{u_{2}} T_{v_{2}}$ if and only if one of the following statements holds:

(a) $u_{1}, u_{2}$ or $v_{1}, v_{2}$ are constants and $u_{1} v_{1}=u_{2} v_{2}$.

(b) $u_{1}$ or $u_{2}$ and $v_{1}$ or $v_{2}$ are not constant and there are some constants $c_{1}, c_{2}, c_{3}, c_{4}$ satisfying $\left|c_{1}\right|+\left|c_{2}\right| \neq 0$ such that

$$
\begin{aligned}
c_{1} u_{1}+c_{2} u_{2} & =c_{3}, \\
c_{1} v_{2}+c_{2} v_{1} & =c_{4}, \\
u_{1} v_{1} & =u_{2} v_{2} .
\end{aligned}
$$

Proof. The sufficiency is easy to check and in what follows we prove the necessity:

(a) If $u_{1}$ and $u_{2}$ both are constants, then $u_{1} v_{1}-u_{2} v_{2}$ is a harmonic function, so $T_{u_{1}} T_{v_{1}}=T_{u_{2}} T_{v_{2}}$ becomes $T_{u_{1} v_{1}}=T_{u_{2} v_{2}}$ which gives $u_{1} v_{1}=u_{2} v_{2}$. In a similar argument, $v_{1}$ and $v_{2}$ are both constants.

(b) Suppose that $v_{1}$ is not constant and one of $u_{1}$ and $u_{2}$ is not constant. So, $v_{1} \notin \mathscr{P}$. It follows from Proposition 5 that

$$
u_{1}=\alpha u_{2}+\beta
$$

for some constants $\alpha$ and $\beta$. So,

$$
0=T_{u_{1}} T_{v_{1}}-T_{u_{2}} T_{v_{2}}=T_{u_{2}} T_{\alpha v_{1}-v_{2}}+T_{1} T_{\beta v_{1}} .
$$

Now, if $\alpha v_{1}-v_{2}$ is not constant, which means $\alpha v_{1}-v_{2} \notin$ $\mathscr{P}$, then, by Proposition 5 again, we get that $u_{2}$ is constant.
By (40), we see that $u_{1}$ is also constant, which is a contradiction. So, $\alpha v_{1}-v_{2}$ is constant, which combined with (40) and (41) gives (39).

Suppose that $v_{2}$ is not constant and one of $u_{1}$ and $u_{2}$ is not constant; then, similar arguments will give (39). The proof is complete.

By (9) and the above result, we can easily get the following two corollaries which have been proved using different methods in [14] and [15], respectively.

Corollary 7. Let $u, v \in \mathscr{M} \cap \mathscr{D}_{h}$. Then, the following statements are equivalent:

(a) $T_{u} \Gamma_{v}=\Gamma_{v} T_{u}$.

(b) $T_{u} T_{J v}=T_{J v} T_{J u}$.

(c) $u$ is constant, or $u$ is not constant, and there are constants $\alpha, \beta$ such that $v=\alpha J u+\beta$ and $(\alpha u+\beta)(u-$ $J u)=0$.

Corollary 8. Let $u, v \in \mathscr{M} \cap \mathscr{D}_{h}$. Then, the following statements are equivalent:

(a) $\Gamma_{u} \Gamma_{v}=\Gamma_{v} \Gamma_{u}$.

(b) $T_{u} T_{J v}=T_{v} T_{J u}$

(c) $v$ is constant and $v(u-J u)=0$, or $v$ is not a constant, and there are constants $\alpha, \beta$ such that $u=\alpha v+\beta$ and $\beta(v-J v)=0$.

As another application of Proposition 5, we have the following.

Corollary 9. Let $u, v, h \in \mathscr{M} \cap \mathscr{D}_{h}$; then, $\Gamma_{u} \Gamma_{v}=\Gamma_{h}$ if and only if $T_{J u} T_{v}=\Gamma_{h}$ if and only if $u=h=0$ or $v=h=0$.

Now, we generalize the above three corollaries to the cases for the general symbols. First, we characterize the commutativity of Toeplitz and Hankel operators.

Theorem 10. Let $U, V \in \mathscr{M}$. Then, $T_{U} \Gamma_{V}=\Gamma_{V} T_{U}$ if and only if $T_{U} T_{J V}=T_{J V} T_{J U}$ if and only if one of the following statements holds:

(a) If $U \notin \mathscr{P}$, then there are constants $\alpha, \beta$ such that $v=$ $\alpha J u+\beta,(\alpha u+\beta)(u-J u)=0$ and, for each $\varphi \in \mathscr{D}_{h}$,

$$
\begin{aligned}
\int_{\mathbb{D}}[U Q(J V \varphi)-J V Q(J U \varphi)] d A \\
\quad+[u-u(0)] \int_{\mathbb{D}}(J V-\alpha J U-\beta) \varphi d A=0 .
\end{aligned}
$$

(b) If $V \notin \mathscr{P}$, then there are constants $\alpha, \beta$ such that $u=$ $\alpha J v+\beta, \alpha J v(v-J v)=0$ and, for each $\varphi \in \mathscr{D}_{h}$,

$$
\begin{aligned}
& \int_{\mathbb{D}}[U Q(J V \varphi)-J V Q(J U \varphi)] d A \\
& \quad+[J v-J v(0)] \int_{\mathbb{D}}(\alpha J V-J U+\beta) \varphi d A=0 .
\end{aligned}
$$


(c) If $U, V \in \mathscr{P}$, then $J v(u-J u)=0$ and, for each $\varphi \in \mathscr{D}_{h}$,

$$
\int_{\mathbb{D}}[U Q(J V \varphi)-J V Q(J U \varphi)] d A=0 .
$$

Proof. First, we prove the necessity. Note that by Proposition 5 we have $u J v-J v J u \in \Delta_{0}$, which means $J v(u-J u)=0$.

(a) If $U \notin \mathscr{P}$, then by Proposition 5 there are constants $\alpha, \beta$ such that $v=\alpha J u+\beta$; this combines with $J v(u-J u)=$ 0 to get $(\alpha u+\beta)(u-J u)=0$. So, by Corollary 7, we have $T_{u} T_{J v}=T_{J v} T_{J u}$. Now, by (23), we have

$$
\begin{aligned}
0= & \left(T_{U} T_{J V}-T_{J V} T_{J U}\right) \varphi \\
= & \left(T_{U} T_{J V}-T_{J V} T_{J U}\right) \varphi(0) \\
& +[u-u(0)] \int_{\mathbb{D}} J(V-v) \varphi d A \\
& -[J v-J v(0)] \int_{\mathbb{D}} J(U-u) \varphi d A
\end{aligned}
$$

for each $\varphi \in \mathscr{D}_{h}$, which combined with $v=\alpha J u+\beta$ will give (a) because

$$
\begin{aligned}
& \left(T_{U} T_{J V}-T_{J V} T_{J U}\right) \varphi(0) \\
& \quad=\int_{\mathbb{D}}[U Q(J V \varphi)-J V Q(J U \varphi)] d A
\end{aligned}
$$

and $\alpha(u-J \mathrm{u})=0$.

(b) If $V \notin \mathscr{P}$, then by Proposition 5 there are constants $\alpha, \beta$ such that $u=\alpha J v+\beta$; this combines with $J v(u-J u)=0$ to get $\alpha J v(v-J v)=0$. So, by Corollary 7, we have $T_{u} T_{J v}=T_{J v} T_{J u}$. The left proof is similar to (a).

(c) Notice that, by Lemma $1, u J v-J v J u \in \Delta_{0}$ means

$$
T_{u J v-J v J u} \varphi=T_{u J v-J v J u} \varphi(0)
$$

for each $\varphi \in \mathscr{D}_{h}$, so combining with (29), (33), and (46), we can get (c) easily.

The sufficiency is obvious by the above arguments. We complete the proof.

With similar and easier arguments, we can get the characterization for commuting of two Hankel operators.

Theorem 11. Let $U, V \in \mathscr{M}$. Then, $\Gamma_{U} \Gamma_{V}=\Gamma_{V} \Gamma_{U}$ if and only if $T_{U} T_{J V}=T_{V} T_{J U}$ if and only if one of the following statements holds:

(a) If $U \notin \mathscr{P}$, then there are constants $\alpha, \beta$ such that $v=$ $\alpha u+\beta, \beta(u-J u)=0$ and, for each $\varphi \in \mathscr{D}_{h}$,

$$
\begin{aligned}
\int_{\mathbb{D}}[U Q(J V \varphi)-V Q(J U \varphi)] d A \\
\quad+[u-u(0)] \int_{\mathbb{D}}(J V-\alpha J U-\beta) \varphi d A=0 .
\end{aligned}
$$

(b) If $V \notin \mathscr{P}$, then there are constants $\alpha, \beta$ such that $u=$ $\alpha v+\beta, \beta(v-J v)=0$ and, for each $\varphi \in \mathscr{D}_{h}$,

$$
\begin{aligned}
\int_{\mathbb{D}}[U Q(J V \varphi)-V Q(J U \varphi)] d A \\
\quad+[v-v(0)] \int_{\mathbb{D}}(\alpha J V-J U+\beta) \varphi d A=0 .
\end{aligned}
$$

(c) IfU, $V \in \mathscr{P}$, then $u J v-v J u \in \Delta_{0}$ and, for each $\varphi \in \mathscr{D}_{h}$,

$$
\int_{\mathbb{D}}[U Q(J V \varphi)-V Q(J U \varphi)] d A=0 .
$$

Now, we consider when the product of two Hankel operators equals another Hankel operator.

Theorem 12. Let $U, V, H \in \mathscr{M}$. Then, $\Gamma_{U} \Gamma_{V}=\Gamma_{H}$ if and only if $T_{J U} T_{V}=\Gamma_{H}$ if and only if one of the following statements holds:

(a) $U, H \in \Delta_{0}$ and, for each $\varphi \in \mathscr{D}_{h}$,

$$
\int_{\mathbb{D}}[J U Q(V \varphi)-J H \varphi] d A=0 .
$$

(b) $V, H \in \Delta_{0}$ and, for each $\varphi \in \mathscr{D}_{h}$,

$$
J U \int_{\mathbb{D}} V \varphi d A-\int_{\mathbb{D}} J H \varphi d A=0 .
$$

Proof. First, assume $T_{J U} T_{V}=\Gamma_{H}$. Then, by Proposition 5, we have Juv $\in \Delta_{0}$ and $h=0$, and the former one means that $J u v=0$, so $u=0$ or $v=0$.

If $u=h=0$, then $U, H \in \Delta_{0}$. In this case, by Lemma 1, $T_{I U} T_{V} \varphi=\Gamma_{H} \varphi$ for each $\varphi \in \mathscr{D}_{h}$ which gives (a).

If $v=h=0$, then $V, H \in \Delta_{0}$. In this case, by Lemma 1, $T_{J U} T_{V} \varphi=\Gamma_{H} \varphi$ for each $\varphi \in \mathscr{D}_{h}$ which gives (b).

The converse is obvious. We complete the proof.

Since $U V \in \Delta_{0}$ with $U$ or $V$ in $\Delta_{0}$, then the following is an easy consequence of the above result which gives the semicommutativity of two Hankel operators.

Corollary 13. Let $U, V \in \mathscr{M}$. Then, $\Gamma_{U} \Gamma_{V}=\Gamma_{U V}$ if and only if $T_{J U} T_{V}=\Gamma_{U V}$ if and only if one of the following statements holds:

(a) $U \in \Delta_{0}$ and, for each $\varphi \in \mathscr{D}_{h}$,

$$
\int_{\mathbb{D}}[J U Q(V \varphi)-J U J V \varphi] d A=0 .
$$

(b) $V \in \Delta_{0}$ and, for each $\varphi \in \mathscr{D}_{h}$,

$$
J U \int_{\mathbb{D}} V \varphi d A-\int_{\mathbb{D}} J U J V \varphi d A=0 .
$$




\section{Essentially Commuting Toeplitz and Hankel Operators}

Recall that Lemma 1 shows that, for $\varphi \in \Delta_{0}, T_{\varphi}$ is at most rank one. It is also the same case for $\Gamma_{\varphi}$ when $\varphi \in \Delta_{0}$ by (9). Moreover, if $U, V \in \mathscr{M}$ and $u, v$ are the Poisson extensions of $\left.U\right|_{\partial \mathbb{D}}$ and $\left.V\right|_{\partial \mathbb{D}}$, respectively, then it is easy to see that $U V=$ $u v+h$ with $h \in \Delta_{0}$. So, $T_{U V}=T_{u v}+F$ with $F$ being a finite rank operator, so by (29) and (33) we have the following result which is proved in [13].

Lemma 14. Let $\varphi, \psi \in \mathscr{M}$ and $\varphi \psi \in \mathscr{M}$; then, $T_{\varphi} T_{\psi}-T_{\psi} T_{\varphi}$ and $T_{\varphi} T_{\psi}-T_{\varphi \psi}$ are both finite rank operators.

Now, we can obtain the conclusions about the compact or finite rank product of Toeplitz and Hankel operators.

Theorem 15. Let $\varphi, \psi \in M$; then, the following statements are equivalent:

(1) $T_{\varphi} \Gamma_{\psi}-\Gamma_{\psi} T_{\varphi}$ is compact.

(2) $T_{\varphi} \Gamma_{\psi}-\Gamma_{\psi} T_{\varphi}$ is finite rank.

(3) $T_{\varphi} \Gamma_{\psi}-\Gamma_{\varphi \psi}$ is compact.

(4) $T_{\varphi} \Gamma_{\psi}-\Gamma_{\varphi \psi}$ is finite rank.

(5) $\psi \in \Delta_{0} \operatorname{or} \varphi-J \varphi \in \Delta_{0}$.

Proof. First, note that, by (9), we have

$$
\Gamma_{\psi} T_{\varphi}-\Gamma_{\varphi \psi}=J\left(T_{\psi} T_{\varphi}-T_{\varphi \psi}\right),
$$

so $\Gamma_{\psi} T_{\varphi}-\Gamma_{\varphi \psi}$ is always finite rank by Lemma 14 . It follows from

$$
T_{\varphi} \Gamma_{\psi}-\Gamma_{\psi} T_{\varphi}=\left(T_{\varphi} \Gamma_{\psi}-\Gamma_{\varphi \psi}\right)-\left(\Gamma_{\psi} T_{\varphi}-\Gamma_{\varphi \psi}\right)
$$

that $(1) \Leftrightarrow(3)$ and $(2) \Leftrightarrow(4)$.

In addition,

$$
\begin{aligned}
T_{\varphi} \Gamma_{\psi}-\Gamma_{\varphi \psi} & =T_{\varphi} T_{J \psi} J-T_{J(\varphi \psi)} J=\left(T_{\varphi} T_{J \psi}-T_{J \varphi J \psi}\right) J \\
& =\left(T_{\varphi} T_{J \psi}-T_{\varphi J \psi}\right) J+T_{\varphi J \psi-J \varphi J \psi} J .
\end{aligned}
$$

Again, by Lemma 14, we see that $T_{\varphi} \Gamma_{\psi}-\Gamma_{\varphi \psi}$ is compact or finite rank if and only if $T_{\varphi J \psi-J \varphi J \psi}$ is compact or finite rank; the latter one is equivalent to $\varphi J \psi-J \varphi J \psi \in \Delta_{0}$ by (14). Since $\varphi J \psi-J \varphi J \psi \in \Delta_{0}$ means $\varphi-J \varphi \in \Delta_{0}$ or $J \psi \in \Delta_{0}$, we get (5). The proof is complete.

Theorem 16. Let $\varphi, \psi, h \in M$; then, the following statements are equivalent:

(1) $\Gamma_{\varphi} \Gamma_{\psi}-\Gamma_{h}$ is compact.

(2) $\Gamma_{\varphi} \Gamma_{\psi}-\Gamma_{h}$ is finite rank.

(3) $T_{\varphi} \Gamma_{\psi}-T_{h}$ is compact.

(4) $T_{\varphi} \Gamma_{\psi}-T_{h}$ is finite rank.

(5) $\varphi, h \in \Delta_{0}$ or $\psi, h \in \Delta_{0}$.
Proof. By (9), we have

$$
T_{\varphi} \Gamma_{\psi}-T_{h}=J\left(\Gamma_{\varphi} \Gamma_{\psi}-\Gamma_{h}\right),
$$

so $(1) \Leftrightarrow(3)$ and $(2) \Leftrightarrow(4)$. In addition,

$$
\begin{aligned}
\Gamma_{\varphi} \Gamma_{\psi}-\Gamma_{h} & =\Gamma_{\varphi} J T_{\psi}-\Gamma_{h}=T_{J \varphi} T_{\psi}-\Gamma_{h} \\
& =\left(T_{J \varphi} T_{\psi}-T_{J \varphi \psi}\right)+\left(T_{J \varphi \psi}-\Gamma_{h}\right) .
\end{aligned}
$$

So, by Lemma $14, \Gamma_{\varphi} \Gamma_{\psi}-\Gamma_{h}$ is compact or finite rank if and only if $T_{J \varphi \psi}-\Gamma_{h}$ is compact or finite rank, and the latter is equivalent to $J \varphi \psi, h \in \Delta_{0}$ by Lemma 2. Since $J \varphi \psi \in$ $\Delta_{0}$ means $J \varphi \in \Delta_{0}$ or $\psi \in \Delta_{0}$, we get (5). The proof is complete.

The following is the easy conclusion of the above result.

Corollary 17. Let $\varphi, \psi \in \mathscr{M}$; then, the following statements are equivalent:

(1) $\Gamma_{\varphi} \Gamma_{\psi}-\Gamma_{\varphi \psi}$ is compact.

(2) $\Gamma_{\varphi} \Gamma_{\psi}-\Gamma_{\varphi \psi}$ is finite rank.

(3) $T_{\varphi} \Gamma_{\psi}-T_{\varphi \psi}$ is compact.

(4) $T_{\varphi} \Gamma_{\psi}-T_{\varphi \psi}$ is finite rank.

(5) $\varphi \in \Delta_{0}$ or $\psi \in \Delta_{0}$.

\section{Conflicts of Interest}

The authors declare that they have no conflicts of interest.

\section{Acknowledgments}

The first and the third authors were supported by NSFC (11671065). The second author was supported by NSFC (11471113) and ZJNSFC (LY14A010013, LY13A010021). This work was started during the first and the second authors' visit to the Department of Mathematics and Statistics, State University of New York at Albany, in the year 2014. They wish to express their great gratitude to the institution for hospitality and Professors Kehe Zhu and Rongwei Yang for helpful discussions.

\section{References}

[1] A. Brown and P. R. Halmos, "Algebraic properties of Toeplitz operators," Journal fur die Reine und Angewandte Mathematik, vol. 213, pp. 89-102, 1964.

[2] P. Ahern and Ž. Čučković, "A theorem of Brown-Halmos type for Bergman space Toeplitz operators," Journal of Functional Analysis, vol. 187, no. 1, pp. 200-210, 2001.

[3] S. Axler and Ž. Čučković, "Commuting Toeplitz operators with harmonic symbols," Integral Equations and Operator Theory, vol. 14, no. 1, pp. 1-12, 1991.

[4] S. Axler and D. Zheng, "Compact operators via the Berezin transform," Indiana University Mathematics Journal, vol. 47, no. 2, pp. 387-400, 1998.

[5] B. R. Choe and Y. J. Lee, "Commuting Toeplitz operators on the harmonic Bergman space," Michigan Mathematical Journal, vol. 46, no. 1, pp. 163-174, 1999. 
[6] Y. J. Lee, "Algebraic properties of Toeplitz operators on the Dirichlet space," Journal of Mathematical Analysis and Applications, vol. 329, no. 2, pp. 1316-1329, 2007.

[7] X. Ding and D. Zheng, "Finite rank commutator of Toeplitz operators or hankel operators," Houston Journal of Mathematics, vol. 34, no. 4, pp. 1099-1119, 2008.

[8] X. Ding, "The finite rank perturbations of the product of Hankel and Toeplitz operators," Journal of Mathematical Analysis and Applications, vol. 337, no. 1, pp. 726-738, 2008.

[9] K. Guo and D. Zheng, "Essentially commuting Hankel and Toeplitz operators," Journal of Functional Analysis, vol. 201, no. 1, pp. 121-147, 2003.

[10] K. Guo and D. Zheng, "Toeplitz algebra and Hankel algebra on the harmonic Bergman space," Journal of Mathematical Analysis and Applications, vol. 276, no. 1, pp. 213-230, 2002.

[11] R. A. Martínez-Avendaño, "When do Toeplitz and Hankel operators commute?" Integral Equations and Operator Theory, vol. 37, no. 3, pp. 341-349, 2000.

[12] Z. Wu, "Hankel and Toeplitz operators on Dirichlet spaces," Integral Equations and Operator Theory, vol. 15, no. 3, pp. 503$525,1992$.

[13] Y. Chen, Y. J. Lee, and Q. D. Nguyen, "Algebraic properties of Toeplitz operators on the harmonic Dirichlet space," Integral Equations and Operator Theory, vol. 69, no. 2, pp. 183-201, 2011.

[14] L. Feng, W. Ma, and L. Zhao, "Commutativity of Toeplitz operator and small Hankel operator on harmonic Dirichlet space," Journal of Mathematics, vol. 35, pp. 917-926, 2015.

[15] L. Feng and W. Ma, "Product of small Hankel operators on the harmonic Dirichlet space," Journal of Mathematical Study, vol. 46, no. 3, pp. 209-220, 2013.

[16] Y. Chen, "Commuting Toeplitz operators on the Dirichlet space," Journal of Mathematical Analysis and Applications, vol. 357, no. 1, pp. 214-224, 2009.

[17] Y. Chen and N. Q. Dieu, "Toeplitz and Hankel operators with $\mathrm{L}^{\infty}$ symbols on Dirichlet space," Journal of Mathematical Analysis and Applications, vol. 369, no. 1, pp. 368-376, 2010.

[18] D. A. Stegenga, "Multipliers of the Dirichlet space," Illinois Journal of Mathematics, vol. 24, no. 1, pp. 113-139, 1980.

[19] Z. Wu, "Carleson measures and multipliers for Dirichlet spaces," Journal of Functional Analysis, vol. 169, no. 1, pp. 148-163, 1999.

[20] T. Yu, "Toeplitz operators on the Dirichlet space," Integral Equations and Operator Theory, vol. 67, no. 2, pp. 163-170, 2010.

[21] L. Zhao, "Hankel operators on the Dirichlet space," Journal of Mathematical Analysis and Applications, vol. 352, no. 2, pp. 767772, 2009. 


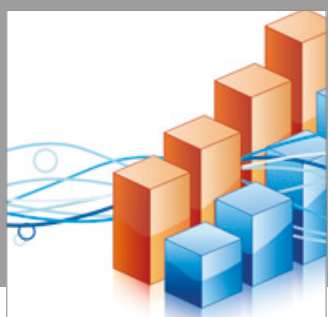

Advances in

Operations Research

vatersals

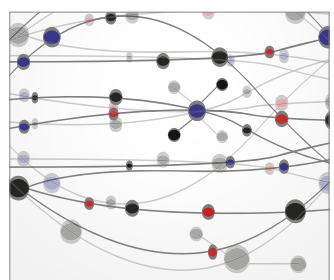

\section{The Scientific} World Journal
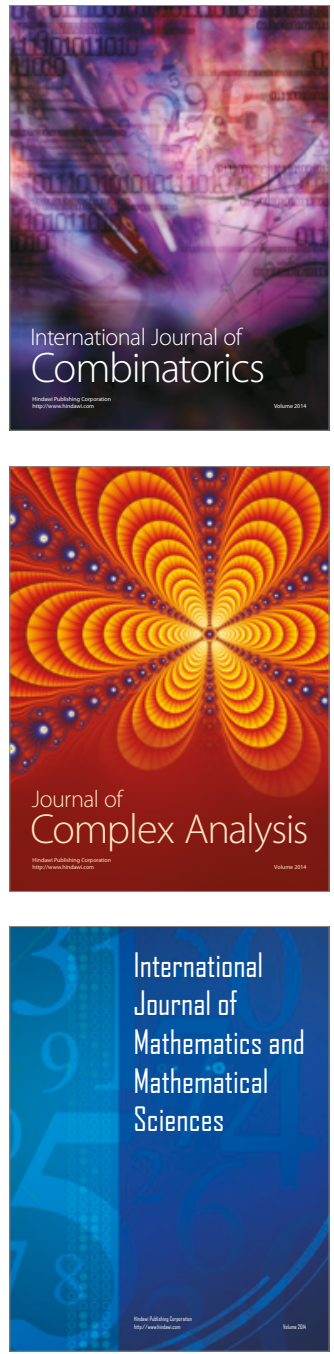
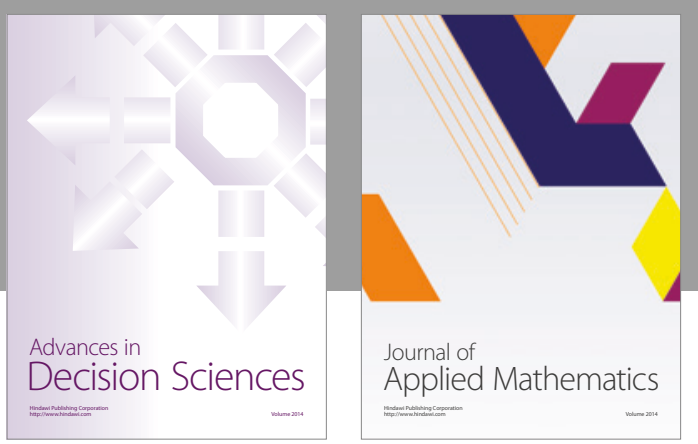

Algebra

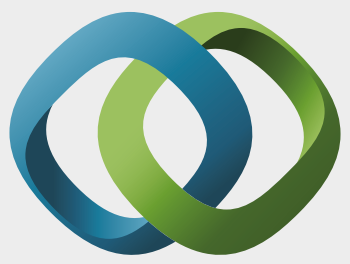

\section{Hindawi}

Submit your manuscripts at

https://www.hindawi.com
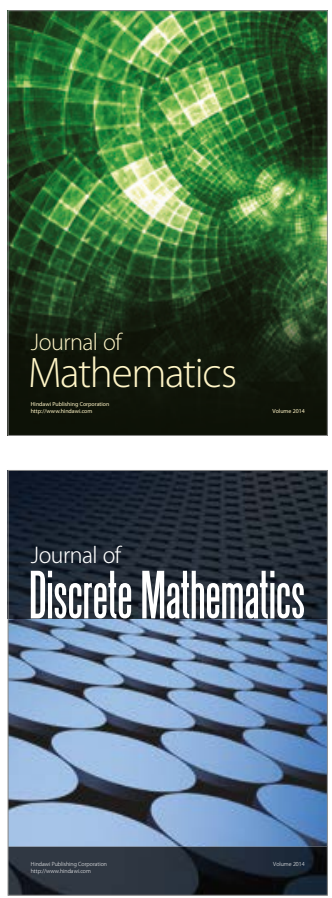

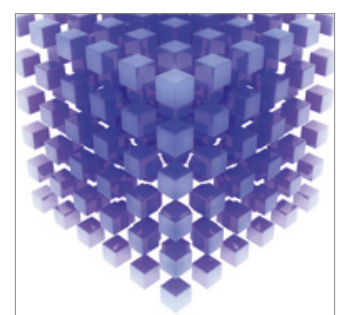

Mathematical Problems in Engineering
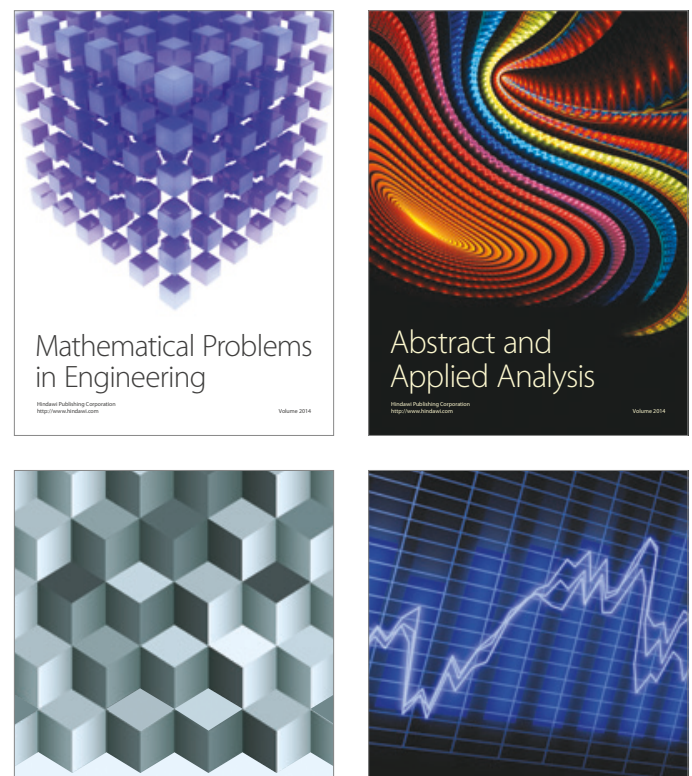

Journal of

Function Spaces

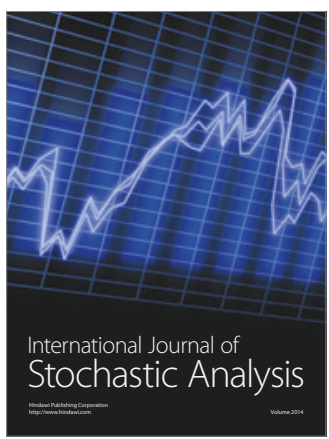

Probability and Statistics
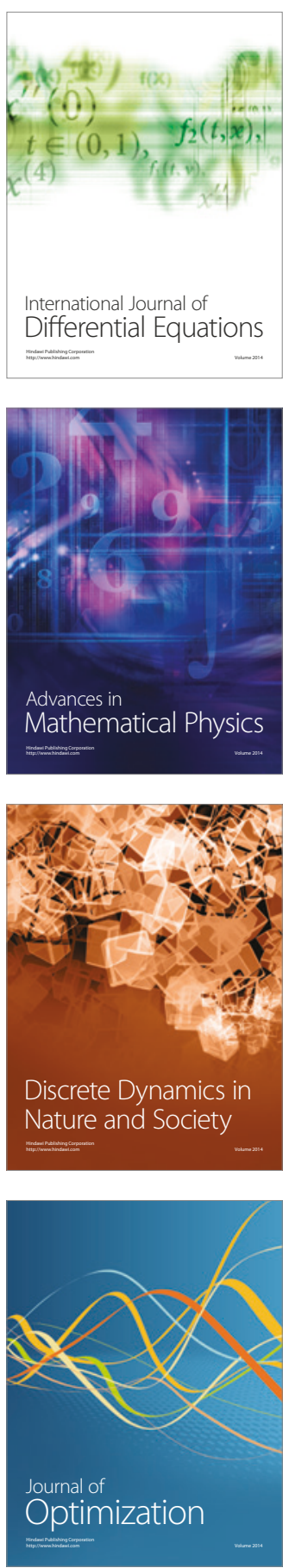